Studies of Solvation Shell Ghanges from the Nuclear Magnetic Resonance Linewidth of ${ }^{23} \mathrm{Na}$

By A. M. Grotzens and J. Smid*

(Chemistry Department, State University of New York, College of Forestry, Syracuse, New York 13210)

and E. DE BOER

(Department of Physical Chemistry, University of Nijmegen, Nijmegen, The Netherlands)

Reprinted from

Chemical Communications 1971

The Chemical Society, Burlington House, London WIV OBN 


\title{
Studies of Solvation Shell Ghanges from the Nuclear Magnetic Resonance Linewidth of ${ }^{23} \mathrm{Na}$
}

\author{
By A. M. Grotews and J. Sum* \\ (Chemistry Department, State University of New York, College of Forestry, Syracuse, New York 13210) \\ and E. DE BOER \\ (Department of Physical Chemistry, University of Nijmegen, Nijmegen, The Netherlands)
}

\begin{abstract}
Summary Addition of polyglycol dimethyl ethers or macrocyclin. polyethers to a solution of sodium tetrainienylboron ion-pairs in tetrahydrofuran strongly broadens the ${ }^{2 N \mathrm{Na}}$ n.m.r. line, indicating that the tetrahydrofuran solvation shell is replaced by the complexing agent.
\end{abstract}

The solvation state of ions or ion-pairs frequently affects their physical properties e.g. their optical and e.s.r. spectra, and reactivity. In mixtures of solvating agents, preferential complexation with the ionic species may occur, and the composition of the solvation shell becomes an important variable. ${ }^{1} \mathrm{H}$ N.m.r. spectra are useful in the identification of the solvent species complexed to an ion or ion-pair, especially when the ionic entity is either paramagnetic ${ }^{1}$ or exhibits strong diamagnetic anisotropy." However, in many cases the proton shifts of the ion solvation shell molecules are small or masked by the solvent.

We report that the addition of small quantities of alkali complexing reagents to solutions of salts such as sodium tetraphenylboride markedly affects the linewidth of the
${ }^{23} \mathrm{Na}$ n.m.r. signal, indicating a change in the solvation shell around the $\mathrm{Na}^{+}$ion. For nuclei with spin quantum number $I>\frac{1}{2}$, particularly for $\mathrm{Na}$ which has a comparatively large quadrupole moment, the linewidth $\left(1 / T_{2}\right)_{Q}$ is largely determined by quadrupole relaxation [equation

$$
\left(\frac{1}{T_{2}}\right)_{0}=\frac{3}{40} \frac{2 I+3}{I^{3}(2 I-1)}\left[\frac{e^{2} Q q}{\lambda}\right]^{2}\left(1+\frac{\kappa^{2}}{3}\right) \tau_{r}
$$

(1) ], ${ }^{3-5}$ where $\tau_{I}$ refers to the rotational correlation time characterizing the tumbling motion of the ion or ion-pair, $e Q$ is the nuclear electric quadrupole moment, eq denotes the largest value of the electric field gradient, and $\kappa$ is the asymmetry parameter.

By combining the expression $\tau_{\mathrm{r}}=4 \pi \eta a^{3} / 3 R T\langle\eta$ is the solution viscbisity and $a$ is the radius of the ionic species approximated by a sphere) with equation (1), equation (2) $(\kappa=0)^{4,8}$ is obtained.

$$
\left(\frac{1}{T_{2}}\right)_{Q} \approx\left(\frac{e^{2} Q q}{\lambda}\right)^{2} a^{3} \frac{\eta}{T}
$$

We measured the linewidth of the ${ }^{29} \mathrm{Na}$ n.m.r. signal of a 
0.2m-tetrahydrofuran (THF) solution of $\mathrm{NaBPh}_{4}$ as a function of temperature, then added $0.2 \mathrm{M}$-hexaethyleneglycol dimethyl ether ( $\mathrm{MeO}\left[\mathrm{CH}_{2} \mathrm{CH}_{2} \mathrm{O}\right]_{6} \mathrm{Me}$ or glyme 7) (I) or 0.2m-dimethyldibenzo-18-crown-6 (II) and repeated the measurements. The ${ }^{23} \mathrm{Na}$ resonance signal was obtained on a Varian DP $60 \mathrm{EL}$ spectrometer at a frequency of $15 \cdot 1 \mathrm{MHz}$, and operating in the high resolution mode. With the crown:compound present the spectrometer was operated in the wide line mode. The chemical shifts were measured with an AEG gaussmeter, using a lM-aqueous $\mathrm{NaCl}$ solution as standard.

Changes in the ${ }^{23} \mathrm{Na}$ chemical shift from 5.7 p.p.m. $\left(\mathrm{NaBPh}_{\mathrm{s}}-\mathrm{THF}\right)$ to 9.2 p.p.m. [NaBPh $-(\mathrm{I})-\mathrm{THF}$ ] to 14.9 p.p.m. [NaBPh 4 (II)-THF] were observed, all shifts being upfield from $\mathrm{NaCl}$ and measured at $40^{\circ} \mathrm{C}$. The shifts are comparable to those found for $\mathrm{NaBPh}_{4}$ in pure tetraglyme and indicate a change in the solvation shell around the $\mathrm{Na}^{+}$ ion. Such alkali n.m.r. shifts have also been reported' for sodium and lithium salts on changing the solvent medium and can be correlated with the solvating ability of the<smiles>COCCOc1cc(C)ccc1OCCOCCOc1ccc(C)cc1OCCOCCO</smiles>

(I)

respective solvents. The Figure shows changes in the linewidths, as $1 / T_{2}$ vs. $\eta / T$ plots. The viscosities, $\eta$, (measured with an Ubbelohde viscometer) are those of the $0.2 \mathrm{M}$-salt solutions in the absence or presence of the complexing agent. The large change in the $\mathrm{Na}$ linewidth of the $\mathrm{NaBPh}_{4}$ salt on addition of an equimolar quantity of $(\mathrm{I})$, and even larger change on addition of (II) is evidence that in the solvent separated $\mathrm{NaBPh}_{4}$ ion-pair the THF solvation shell around the $\mathrm{Na}^{+}$ion is replaced by (I) or by (II). Studies with small quantities of tetraglyme show similar behaviour and addition of more than an equimolar quantity of (I), after correction for the increased viscosity, does not lead to further line broadening, indicating the formation of $1: 1$ complexes between the crown compound or glymes and the $\mathrm{NaBPh}_{\text {. }}$.

Two factors may be responsible for the increased linewidth, viz., a change in the rotational correlation time $\tau_{\mathbf{r}}$ of the ion-pair on changing the solvation shell around the $\mathrm{Na}^{+}$ ion, or a change in the electric field gradient at the Na nucleus. We suggest that the latter factor is the more important one. The overall size of the solvated ion-pair does not change significantly when the THF solvation shell is replaced by either (I) or (II). Viscosities of the $0.2 \mathrm{M}-\mathrm{NaBPh}_{\mathrm{a}}$ solutions are nearly identical in the absence and presence of the complexing agent. However, in the THF-separated ion-pair the oxygen atoms are nearly spherically distributed around the $\mathrm{Na}+$ ion. This is probably not the case for compound (I) and certainly not for (II) which derives its strong alkali complexing property

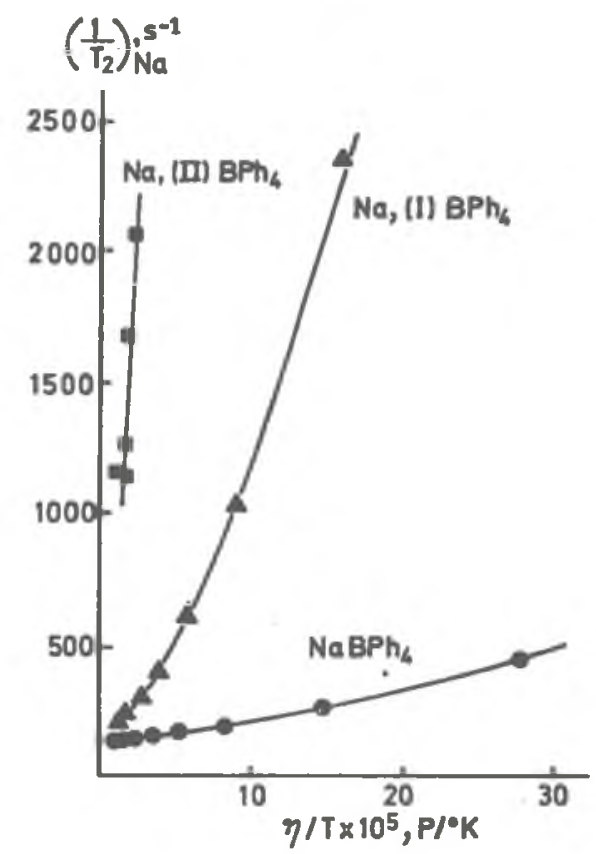

Figure. Changes in the linewidth $1 / \mathrm{T}_{1}$ of the ${ }^{20 \mathrm{Na}}$ n.m.r. signal of sodium tetraphenylboride on addition of equimolar quantities of alkali complexing agents. Concentration of all species:0-2M. For $0.5 \mathrm{M}-\mathrm{NaBPh}$, solutions, the $1 / \mathrm{T}_{2}$ value was found to be the same as for $0.2 \mathrm{M}-\mathrm{NaBPh}_{4}$ at corresponding $\eta / \mathrm{T}$ values.

largely from the fact that the polyether ring can assume a nearly planar conformation, with the $\mathrm{Na}^{+}$ion situated in the hole of the polyether ring. ${ }^{8}$ This non-spherical type of solvation would strongly increase the electric field gradient contributed by the lone electron pairs of the oxygen atoms at the $\mathrm{Na}$ nucleus. The small curvature in the linewidth plots may indicate that slight changes take place in the solvated ion-pair structure as the temperature is lowered, e.g., an increased ordering around the $\mathrm{Na}^{+}$ ion.

This method is useful in the study of ion and-ion-pair solvation, especially in cases where ${ }^{1} \mathrm{H}$ n.m.r. studies do not provide conclusive evidence. Also, the complexation of certain crown compounds with sodium ions or ion-pairs may be slow enough ${ }^{2 b}$ for a kinetic study to be made from the change in the linewidth of the ${ }^{29} \mathrm{Na}$ n.m.r. signal.

We thank the National Institutes of Health and the University of Nijmegen, for financial support.

(Reccived, April 19th, 1971; Com. 596.)

1 A. Fratiello and D. P. Miller, Mol. Phys., 1966, 11, 37; E. de Boer, A. M. Grotens, and J. Smid, Chem. Comm., $1970,1035$.

(a) J. A. Dixon, P. A. Gwinner, and D. C. Lini, J. Amer. Chem. Soc., 1965, 87, 1379; (b) K. H. Wong, G. Konizer, and J. Smid, ibid., $1870,92,666$.

"A. Abragam, "The Principles of Nuclear Magnetism," Clarendon Press, Oxford, 1961, Chap. 8.

- G. Bonera and A. Rigamonti, J. Chem. Phys., 1965, $42,175$.

G G. W. Canters, Ph.D. Thesis, University of Nijmegen, The Netherlands, 1969, p. 26.

- R. H. Erlich, E. Roach, and A. I. Popov, I. Amer. Cheme. Soc., 1970, 92, 4989.

7 C. Carvajal, K. J. Tölle, J. Smid, and M. Szwarc, J. Amer. Chem. Soc., 1965, 87, 5548.

C. J. Pedersen, J. Amer. Chem. Soc., 1967, 89, 7017. 